\title{
Investigating 3D Printing with Microscopy and Spectroscopy Techniques
}

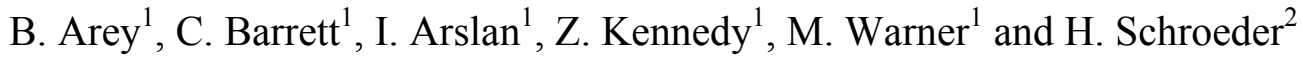 \\ 1. Pacific Northwest Laboratory, 902 Battelle Boulevard, Richland, WA., USA \\ 2. Bruker Nano Analytics, Billerica, MA, USA
}

3D printing, also known as additive manufacturing (AM), turns digital 3D models into solid objects by building them up in layers. The technology was first invented in the 1980s, and since that time has primarily seen use for rapid prototyping (RP). However, in the last few years, 3D printing has started to evolve into a next-generation manufacturing technology that has the potential to allow the local, ondemand production of final products or parts thereof. 3D printing encompasses a wide range of additive manufacturing technologies. Each process builds objects in successive layers, with thicknesses on the order of $0.1 \mathrm{~mm}$. The methods used vary significantly, but all start with a computer aided design (CAD) model or a digital scan. This is then processed by 'slicing software' that divides the object into thin cross sections that are printed out one on top of the other.

Additively manufactured objects are becoming increasingly prevalent across many market sectors, from electronics and transport vehicles to medical devices and implants. Over the last decade, the relatively inexpensive "desktop" 3D printers have quite literately brought manufacturing to the masses. To that end, it is quite feasible to assume that AM will contribute to, if not directly play a role in the counterfeiting of certain products or parts. When one considers the ease in which digital CAD files can be shared, the potential threat of counterfeit products and devices flooding markets is greatly increased. Due to the rapid emergence of more sophisticated AM devices and facilities around the globe, it could be difficult, if not impossible to determine where it was manufactured and by whom, unless we begin to develop a more robust signature set. Today, this type of determination is not possible without developing new tools and protocols that will lead to a better understanding of the intricacies of various AM processes. A thorough understanding of the chemical and materials signatures of these types of objects, as well as the raw materials from which they are produced, will give the ability to make determinations about how an object was manufactured, who manufactured it, and potentially why the object was manufactured.

A main focus for this effort was the evaluation of the usability of micro X-ray Tomography $(\mu \mathrm{CT})$ as a non-destructive analytical tool for metal particle determination. The combination of dedicated specimen preparation with state-of-the-art, X-ray and electron-based microscopic and tomographic techniques proves a powerful methodology to reveal otherwise inaccessible information regarding structural organization over the whole range of length scales. It is expected that these tools will play a crucial role in the rationalization of scale-up principles in forensic development. Combining the $\mu \mathrm{CT}$ and FIB-SEM and TEM is novel concept for 3D printed material. The concept has been used in the geological studies [5]. 


\section{References:}

[1] I. R. Gibson, David W., Stucker, Brent, Additive Manufacturing Technologies: Rapid Prototyping to Direct Digital Manufacturing, (Springer, 2010).

[2] B. Berman, Bus. Horizons 55 (2012), p. 155.

[3] B. N. Turner, R. Strong and S. A. Gold, Rapid Prototyping J. 20 (2014), p. 192.

[4] B. M. Tymrak, M. Kreiger and J. M. Pearce, Mater. Des. 58 (2014), p. 242.

[5] Acknowledgements: This work was supported by the Laboratory Directed Research and Development (LDRD) program at the Pacific Northwest National Laboratory (PNNL) operated by Battelle for the U.S. Department of Energy. Bruker Nano Analytics, Billerica, Ma for the assistance on the $\mu \mathrm{CT}$ scans.
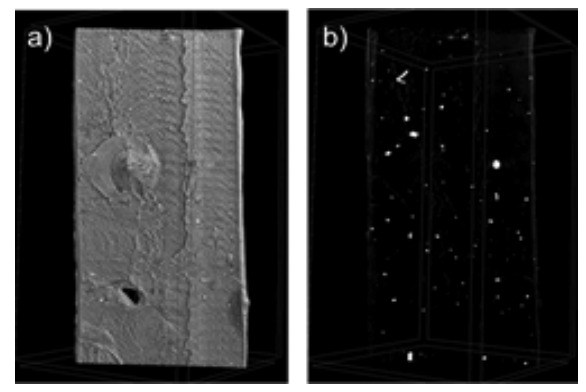

Figure 1. Structural characterization of (85:15)-PVDF/MWCNT $(\mathrm{a}, \mathrm{b})$ with approximate dimensions 5.5 x $2.5 \times 0.4$ (height $(\mathrm{h}) \times$ width $(\mathrm{w}) \times$ thickness $(\mathrm{t}), \mathrm{mm})$ and of commercially available PLA/graphene

\section{BF-DF Pairs}

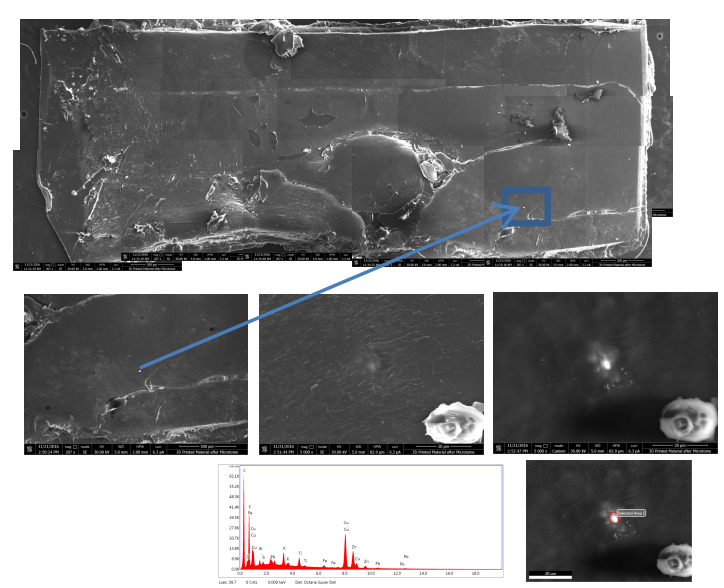

Figure 2. SEM images and EDS spectra

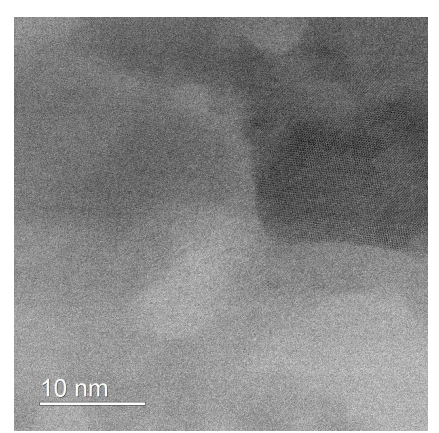

$\mathrm{BF}$

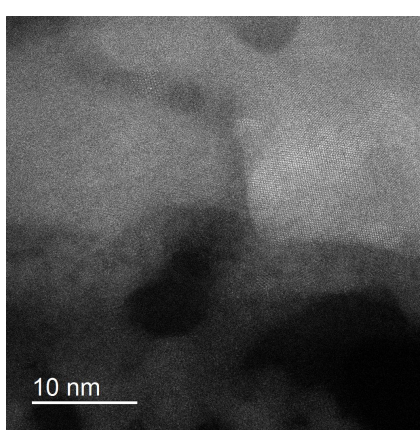

DF

Figure 3. TEM Images 